DOI:

10.1038/nri2109

\title{
Opposing roles for osteopontin
}

An intriguing feature of cytokines is that depending on the time and the place, the same cytokine can have opposite effects. A recent study in Nature Medicine describes how the cytokine osteopontin can have a pro-inflammatory and detrimental effect during the primary phase of allergic airway disease but an antiinflammatory and protective effect during secondary antigenic challenge. These opposing effects of osteopontin in allergic disease seem to be a result of its influence on the recruitment of different subsets of dendritic cells (DCs).

To assess the role of osteopontin in the regulation of $\mathrm{T}$ helper 2 $\left(\mathrm{T}_{\mathrm{H}} 2\right)$-cell-mediated allergic responses, Xanthou et al. used an

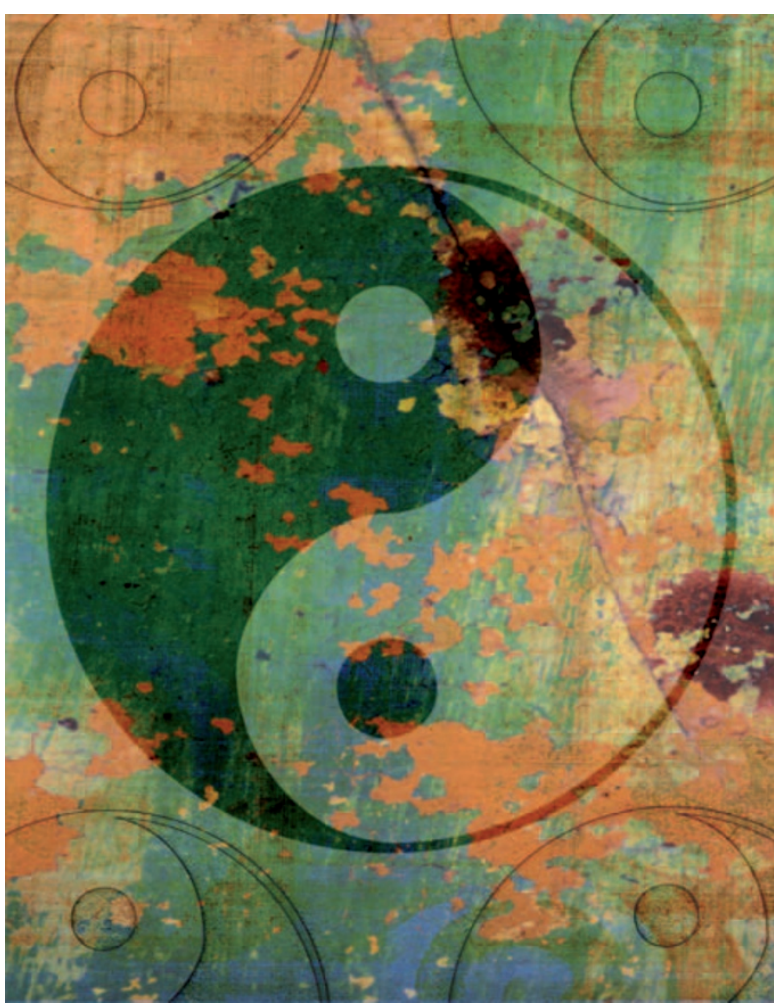

established mouse model of ovalbumin (OVA)-induced allergic airway inflammation. In this model, airway inflammation is induced by sensitization with OVA plus alum followed by airway challenges with OVA. The authors show that if mice were given a neutralizing antibody specific for osteopontin before the sensitization phase, the inflammatory response to subsequent OVA challenges was significantly diminished compared with control mice. Thus, the antibody-treated mice showed decreased airway hyperresponsiveness (AHR), a reduced inflammatory-cell infiltrate (including $\mathrm{T}_{\mathrm{H}} 2$ cells) in the lung-draining lymph nodes and lower levels of $\mathrm{T}_{\mathrm{H}}$ 2-cell-associated cytokines, such as interleukin-4 (IL-4) and IL-13, in lymph-node cultures. By contrast, if osteopontin-specific neutralizing antibody was administered to mice immediately before secondary challenge with OVA, the opposite effect was observed - AHR worsened, the inflammatory-cell infiltrate increased and the levels of $\mathrm{T}_{\mathrm{H}}$ 2-type cytokines were higher. So, neutralization of osteopontin seems to have different effects depending on the stage of the allergic response.

How might this dichotomy be achieved? Plasmacytoid DCs (pDCs) have been shown to have a suppressive effect on $\mathrm{T}_{\mathrm{H}} 2$-cell responses, so the authors tested whether osteopontin blockade at the sensitization phase might affect pDC recruitment. Indeed, compared with control mice, mice treated with osteopontin-specific antibody before sensitization showed increased numbers of pDCs in the draining lymph nodes. Importantly, the depletion of pDCs before sensitization and antibody treatment restored OVA-driven responses, indicating that the decrease in $\mathrm{T}_{\mathrm{H}} 2$-cell priming observed after osteopontin blockade was mediated by increased numbers of pDCs that have a regulatory function. Conversely, during secondary challenge, osteopontin blockade caused a more marked increase in the recruitment of conventional DCs than pDCs to the draining lymph nodes. In co-cultures, conventional DCs from these draining lymph nodes promoted the differentiation of OVA-specific CD4 ${ }^{+}$ $\mathrm{T}$ cells into $\mathrm{T}_{\mathrm{H}} 2$ cells. So, the increased recruitment of immunogenic conventional DCs provides an explanation for the enhanced $\mathrm{T}_{\mathrm{H}}$ 2-cell responses observed after osteopontin blockade at the secondary challenge.

Finally, the authors tested the therapeutic potential of recombinant osteopontin in allergic disease. Consistent with their previous observations, administration of recombinant osteopontin during sensitization had a pro-inflammatory effect, whereas osteopontin administration during the secondary challenge decreased the inflammatory response and conferred protection from allergic disease.

As dysregulated osteopontin expression has been linked to several autoimmune and cardiovascular diseases, osteopontin might be of therapeutic benefit in other disorders, if given at the right stage.

Lucy Bird

ORIGINAL RESEARCH PAPER Xanthou, G. et al. Osteopontin has a crucial role in allergic airway disease through regulation of dendritic cell subsets. Nature Med. 13, 570-578 (2007) FURTHER READING Shinohara, M. L. \& Cantor, H. The bridge between dendritic cells and asthma. Nature Med. 13, 536-538 (2007) 